\title{
Estudo de Caso do Uso da Realidade Aumentada no Setor de EAD do CESUP
}

\section{Case Study of the Use of Augmented Reality in the Distance Education Section of CESUP}

\begin{abstract}
Resumo: Neste artigo é apresentado um estudo de caso sobre o uso da realidade aumentada como ferramenta auxiliar ao vídeo educacional com o objetivo de explorar o potencial desta tecnologia no setor de Educação à distância (EAD) do Centro Nacional de Supercomputação da Universidade Federal do Rio Grande do Sul (CESUP/UFRGS). Para analisar a contribuição da mesma na construção do conhecimento, foram feitos experimentos dentro da estrutura já existente no CESUP com câmeras controladas com joystick e através de uma mesa de apresentação de documentos. Foi produzido um vídeo de caráter exploratório e demonstrativo sobre o uso da Realidade Aumentada (RA) como tecnologia educacional para a criação de uma rede adicional para o Supercomputador do CESUP/ UFRGS. O trabalho compartilha a apresentação de um modelo virtual do cluster do CESUP através de Realidade Aumentada para o público no evento UFRGS Portas Abertas 2012. Os resultados dos experimentos mostram que o emprego da RA na área de educação e treinamento apresenta um grande potencial de auxiliar o processo de construção do conhecimento dos usuários.

Palavras-Chave: Realidade aumentada. Tecnologias educacionais. Educação à distância. Vídeo educacional.
\end{abstract}

\footnotetext{
Abstract: This paper presents a case study on the use of augmented reality as a tool to assist educational video with the aim of exploring the potential of this technology in the sector of the distance education (EAD) of National Center for Supercomputing of the Universidade Federal do Rio Grande do Sul (CESUP/UFRGS). To analyze the contribution of the same in the knowledge construction, experiments were done within the existing structure in CESUP/UFRGS with cameras controlled by joystick and a visual presenter for documents. An experimental video was produced to demonstrate the use of Augmented Reality (AR) as educational technology to create an additional network for Supercomputer in CESUP/UFRGS. The work shares the presentation of a virtual model of the CESUP cluster through Augmented Reality to the public at the event UFRGS Portas Abertas 2012. The results of the experiments showed that the use of AR in education and training has great potential to assist the process of constructing knowledge of users.

Keywords: Augmented reality. Educational technologies. Distance education. Educational video.

SCHNEIDER, Eduardo Luis; GRASSI, André Soares; PICCOLI, Leonardo Bisch; TIMM, Maria Isabel. Estudo de Caso do Uso da Realidade Aumentada no Setor de EAD do CESUP. Informática na Educação: teoria e prática, Porto Alegre, v. 17, n. 2, p. 75-88, jul./dez. 2014.
}

\section{Eduardo Luis Schneider}

André Soares Grassi

Leonardo Bisch Piccoli

Maria Isabel Timm

Universidade Federal do Rio Grande do Sul

\section{Introdução}

0 crescente aumento na complexidade imposta por tarefas em diversas áreas da Ciência está exigindo cada vez mais do homem, frente às suas limitações naturais. Da mesma maneira, o número de trabalhos que incorporaram o computador para o seu desenvolvimento vem crescendo e aumentando, também, a dependência do homem em relação à máquina. Para lidar com esta realidade, surge a necessidade da criação de ambientes mais 
interativos, que permitam que o homem minimize possíveis dificuldades na manipulação do computador e, por conseguinte, execute seu trabalho de modo mais eficiente e satisfatório. Com o uso de novas tecnologias, as atividades e as práticas de ensino se tornam mais favoráveis para o aprendizado, influenciando diretamente em sua qualidade (KLOPFER, 2008).

Através da evolução das tecnologias de mistura da Realidade Virtual (RV) com o mundo real, é possível colaborar com as diversas áreas do conhecimento, tanto das ciências humanas quanto das exatas. Nas engenharias, por exemplo, onde são buscadas soluções para muitos processos industriais de intensa complexidade e que demandam o uso de ferramentas de visualização dos seus sistemas, técnicas de visualização como a RV e a Realidade Aumentada (RA) se apresentam como poderosa ferramenta para visualização tridimensional. O suporte aos processos industriais de produção pode se dar através da sobreposição de objetos virtuais gerados pelo computador ao ambiente real, como partes funcionando em tempo real de um equipamento que precise ser monitorado.

Este trabalho visa desenvolver e explorar o potencial da RA como ferramenta de suporte ao Núcleo de Pesquisa e Desenvolvimento de Tecnologias Educacionais e EAD do Centro Nacional de Supercomputação (CESUP), da Universidade Federal do Rio Grande do Sul (UFRGS). O Centro oferece Processamento de Alto Desempenho (PAD) a usuários e pesquisadores que necessitam resolver problemas avançados e realizar simulações, tanto em ciências básicas como em aplicadas aos mais diversos campos (UNIVERSIDADE FEDERAL DO RIO GRANDE DO SUL, 2011). Um dos serviços prestados é o treinamento dos pesquisadores para o uso do PAD. O Núcleo de Pesquisa e Desenvolvimento de Tecnologias Educacionais e
EAD se encarrega dessa tarefa e também mantém parcerias na pesquisa e uso de tecnologias educacionais.

Este artigo está estruturado de forma a abordar a Realidade Aumentada, as Bibliotecas Gráficas empregadas com esta tecnologia, o uso da Realidade Aumentada em conjunto com o Ensino à Distância. Nos procedimentos metodológicos, inicialmente é feita uma descrição da Infraestrutura de EAD e Multimídia do CESUP/UFRGS. Posteriormente, é detalhada a utilização da RA como Suporte a Criação de uma Virtual LAN (VLAN) no CESUP/UFRGS, onde é produzido um vídeo, com caráter exploratório e demonstrativo, que experimenta a RA com o professor segurando a marcador para a captura por câmeras com controle remoto e a imagem de um switch no monitor, bem como através da utilização de uma mesa de apresentação de documentos. Além disso, o artigo compartilha a apresentação de um modelo virtual do cluster do CESUP através da RA para o público no evento UFRGS Portas Abertas 2012. Na sequencia, o estudo de caso do uso da RA integrada ao setor de EAD do CESUP compara a RA com as outras ferramentas disponíveis através de critérios como custo de produção e usabilidade.

\section{Realidade Aumentada}

A Realidade Aumentada (RA) permite representar dados e informações que não estão diretamente disponíveis ou detectáveis apenas pelos sentidos humanos, e seu emprego possibilita aumento dessa percepção. Assim, pode auxiliar na melhoria da interação com o mundo real e, consequentemente, aumentar a produtividade na realização dos mais variados tipos de tarefas. Entre as áreas de aplicação que já vêm sendo exploradas, podem ser citadas: pu- 
blicidade, jogos, entretenimento, ilusionismo, indústria automobilística, militar, turismo, preservação histórica, mercado imobiliário, medicina, comunicação, movimentação de robôs, manutenção de equipamentos e educação.

Sabe-se que a simulação da realidade está longe de ser comparada ao mundo real conhecido. A mescla de ambientes virtuais e reais apareceu como uma alternativa: a adição de objetos virtuais a uma cena real. Essa área é chamada de Realidade Misturada (RM) e trabaIha com aplicações em que ambientes virtuais e reais são sobrepostos, permitindo aos usuários manter o senso de presença no ambiente onde se encontram, continuando a ver ou sentir os elementos do ambiente real (HULF et al., 2004, AZUMA et al., 2001). Segundo Milgram et al. (1994), na RM, a visão do mundo real é combinada com o modo de visão do Ambiente Virtual ( $A V)$, e as transições entre esses dois pilares dividem a RM em duas subáreas: a Realidade Aumentada (RA) e a Virtualidade Aumentada (VA), conforme ilustra a Figura 1. $\mathrm{Na} V \mathrm{~A}$, o ambiente principal ou predominante é o mundo virtual. Na RA, o que predomina é o mundo real.

Na VA, o AV é realçado por meio do acréscimo de imagens, elementos ou sensações advindas do mundo real, fazendo com que o usuário possa ver seu próprio corpo, por exemplo, como algo presente nas simulações geradas por computador. Outra possibilidade seria inserir uma mobília real em um ambiente virtual criado (SISCOUTO; TORI, 2004, PROVIDELO, 2004).

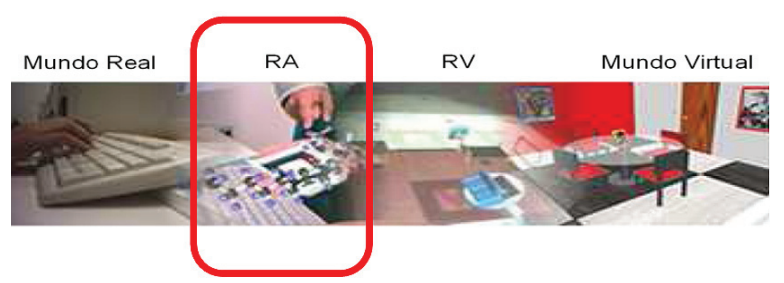

FIGURA 1 - Ambiente de Realidade Misturada Destacando Onde se Situa a Realidade Aumentada.

FONTE: Adaptado de Milgram et al., 1994.

Já a RA pode ser definida como a sobreposição de objetos virtuais ao mundo real, por meio de um dispositivo tecnológico, melhorando ou aumentando a visão do usuário (BAJURA; NEUMANN, 1995, AZUMA, 1997, MILGRAM et al., 1994). Cabe ressaltar que, neste caso, os objetos virtuais são trazidos para o espaço do usuário, onde ele tem segurança e sabe como interagir, sem a necessidade de treinamento. Além disso, o usuário consegue manipular os objetos reais e virtuais do ambiente misturado com o uso das próprias mãos. A RA pode ser empregada para a capacitação de funcionários, inclusive em serviços de risco para a aplicação ou até para o próprio funcionário. O usuário pode obter informações a respeito de um determinado equipamento danificado e ser guiado para a solução do problema a partir da adição de informações virtuais ao ambiente real onde o equipamento está.

$\mathrm{O}$ rastreamento, em ambientes de $\mathrm{RV}$ e RA, tem a função de identificar a posição do usuário ou de algo atrelado a ele, e permite o controle de posicionamento em ambientes virtuais ou aumentados. Diferentes dispositivos de rastreamento foram desenvolvidos, usando princípios mecânicos, magnéticos, de ultrassom, ótico, etc. O rastreamento ótico, junto com a popularização da webcam e o aumento do poder de processamento dos microcomputadores, está fazendo com que a RA esteja se 
apresentando como uma possibilidade concreta de vir a ser a próxima geração de interface popular.

\subsection{Bibliotecas Gráficas}

Para o desenvolvimento de aplicações de RA é necessário utilizar algumas bibliotecas gráficas. A mais conhecida atualmente é o ARToolKit que, além de apresentar resultados adequados, é gratuita e de código aberto (ARTOOLKIT, 2005). Existem outras, como o ARTag (FIALA , 2005, ARTAG, 2010), que estão sendo desenvolvidas com propósitos comerciais.

O ARToolKit é um software desenvolvido pelo Dr. Hirozaku Kato da Universidade de Osaka, no Japão, e apoiado pelo Human Interface Technology Laboratory da Universidade de Washington e de Canterbury na Nova Zelândia. Foi projetado para o desenvolvimento de aplicativos de RA, provendo técnicas de visão computacional para calcular a posição e a orientação de marcadores impressos e capturados por uma câmera digital ou um Head Mounted Display (HMD), de forma que os marcadores venham a ser revestidos por objetos virtuais 3D. Utiliza-se de técnicas de visão computacional para calcular o ponto de vista da câmera em relação a um marcador existente no mundo real. Cada quadro de imagem do ambiente capturado é binarizado para facilitar o reconhecimento e a posição espacial do marcador. $O$ desenho no interior do marcador reconhecido é comparado com marcadores pré-definidos, e assim o marcador é sobreposto por uma imagem. A imagem gerada pode ser de um objeto desenvolvido em bibliotecas gráficas como OpenGL, ou importado em linguagem Virtual Reality Modeling Language (VRML). O VRML é uma linguagem para mode- lagem em RV. Seu objetivo é a criação e representação de mundos tridimensionais e objetos interativos através da WWW (World Wide Web) na internet. Foi apresentada pela primeira vez em 1994 durante a conferência sobre WWW com o propósito de atingir tais objetivos sem necessitar de uma rede de alta velocidade para dar suporte às atividades (CRISPEN, 2010).

Para facilitar o desenvolvimento de aplicações colaborativas de realidade aumentada para usuários não especialistas em computação, foi desenvolvido o Sacra, que é uma ferramenta de autoria, onde as atividades de configuração podem realizadas utilizando pastas e arquivos de texto, elaborados com o Bloco de Notas do Windows ou equivalente (KIRNER; SANTIN, 2010).

\subsection{Realidade Aumentada e Ensino à Distância}

Ambientes de Realidade Virtual e Aumentada (RVA) implementados em rede ampliam em muito o potencial de aplicações educacionais. $O$ estudo da RA em ambientes colaborativos proporciona compartilhamento de objetos virtuais no espaço real, de modo que todos os usuários possam ter a mesma sensação de espaço, presença e tempo (KIRNER, 2005).

Um interessante estudo sobre máquinas elétricas auxiliado por técnicas de RV é mostrado em Bernardino A. Junior, Cardoso e Lamounier (2000). Outro estudo relacionado com RA foi sobre o desenvolvimento de uma interface gráfica para o ARToolKit, com aplicação na área educacional (CUNHA; GUIMARÃES, 2007). Em Trigo e Kirner (2007) são exemplificados mais alguns trabalhos sobre essa abordagem, como o de uma aplicação para treinamento industrial colaborativo baseado em RA distribuída. Tam- 
bém é apresentado o desenvolvimento de um Ambiente Virtual de Aprendizagem, denominado AVADPT, que contém módulos de realidade virtual e realidade aumentada. O ambiente criado é adaptativo, de acordo com o grau de familiaridade do professor com a tecnologia, e pode ser usado separadamente ou em conjunto com outros ambientes educacionais, como o Moodle.

Uma interessante área de treinamento é a de manutenção de equipamentos, principalmente em processos industriais, pois nesses casos os ambientes tradicionais de treinamento apresentam limitações e alto custo (BLUEMEL et al., 2003). Tecnologias RVA podem permitir a visualização e manipulação de componentes de difícil acesso, além de oferecer um suporte nas operações de montagem e desmontagem de equipamentos, nem sempre previstas em manuais. A utilização de interfaces para exibir as informações de suporte à manutenção cria uma maior interatividade do usuário com o equipamento em que é necessário realizar alguma intervenção. Alguns requisitos a respeito de treinamento virtual são apresentados em Boud et al. (1999) e Li; Khoo; Tor (2003).

Uma aplicação interessante foi desenvolvida por pesquisadores do grupo Steve Feiner, da Universidade de Columbia. Ela ajuda usuários no processo de manutenção de uma impressora laser (FEINER; MACINIYRE; SELIGMANN, 1993). Este projeto usa rastreadores de posição localizar a parte dessa impressora na qual é necessário realizar uma operação. Além disso, assinala a presença ou não de algum objeto obstruindo o caminho de uma determinada ação que, concluída, eliminaria a necessidade do modelo virtual.

Para esses propósitos, equipamentos de visualização como TabletPC, Personal Digital Assistant (PDA), HMD e outros podem ser usados
(COMPORT; MARCHAND; CHAUMETTE, 2003). O usuário também pode receber informações do sistema de manutenção, como gráficos 2D, modelos 3D e guias textuais de informações de manutenção. Outra possibilidade é a interação por comando de voz, especificando-se os componentes do equipamento e o tipo de informação que se deseja visualizar (ESPÍNDOLA et al., 2010). Diversos outros trabalhos poderiam ser citados aqui. Contudo, não foi objetivo deste trabalho consolidar ou contrapor o estado da arte na área de RA, mas sim mostrar um direcionamento que está sendo dado no CESUP/UFRGS.

\section{Infra-estrutura de EAD e Multimí- dia do CESUP/UFRGS}

Desde sua implantação em 2007, a infraestrutura de produção de aulas à distância e multimídia educacional do CESUP/UFRGS tem servido ao treinamento dos usuários do cluster (conjunto de computadores com sistema operacional distribuído) Sun Fire, mas igualmente à pesquisa e parcerias na área de tecnologias educacionais. Conforme descrito por Timm et al. (2008, p. 3.), o conjunto pretende ser um "[...] sistema permanentemente instalado (plug-and-play) de produção, edição e transmissão de eventos interativos [...]", priorizando

[...] a criação de condições para ministrar cursos que demandem alta exigência de visualização, ou que possam ser beneficiados pelos recursos audiovisuais em multimídia, para apoio à compreensão de conteúdos exigentes e complexos, como a computação de alto desempenho, e outras áreas científicas e tecnológicas, como engenharia e medicina [...] (TIMM et al., 2008, p. 3) 
A infraestrutura montada no Núcleo de Pesquisa e Desenvolvimento de Tecnologias Educacionais e EAD do CESUP dispõe de sala de aula (e estúdio de gravação) com isolamento acústico e iluminação adequada; duas câmeras com movimento e zoom remotamente controlados por joystick para captação do ambiente; uma mesa de apresentação de documentos, dotada de câmera de vídeo própria e iluminadores orientados a 450; um computador de uso do professor com saídas de vídeo e áudio conectadas ao sistema; e uma ilha de captação/edição, que possibilita escolher, em tempo real, entre as quatro fontes de vídeo mencionadas. Todos esses recursos estão instalados de forma permanente, tendo em vista a agilidade e funcionalidade, mas também permitindo a integração entre si e com outras tecnologias a serem acrescentadas. É assim que se está experimentando a Realidade Aumentada. Através da técnica, o professor cuja aula está sendo gravada e/ou transmitida pode apresentar uma visualização 2D ou 3D de determinado objeto, componente ou cena, como se estivesse em sua mão, da mesma forma que faria com um livro aberto em que se apresentasse uma ilustração. Neste caso, porém, a imagem é integrada com a referida estrutura de captação e edição de vídeo, podendo ser visualizada em detalhe por cada aluno, presencial e/ou à distância, em tempo real ou através de acesso posterior ao acervo de vídeo-aulas.

Pesquisa-se também a possibilidade de utilização remota da Realidade Aumentada, pelos alunos à distância: o acesso a determinado conteúdo de vídeo, áudio e/ou 3D com o próprio computador do estudante. Desta forma, se experimentaria laboratórios virtuais de baixo custo e sem grande estrutura remota, conforme descrito por Forte et al.:
O usuário geralmente observa a ocorrência de eventos a partir da tela do computador, sem a necessidade de se munir de dispositivos especiais de visualização, e interage com os objetos virtuais com o auxílio de marcadores tangíveis, sem a necessidade, também, de dispositivos especiais para a tarefa. (FORTE et al., 2008, p.24)

\section{Utilização da RA Como Suporte a Criação de uma VLAN}

A integração da RA à estrutura já existente no CESUP/UFRGS está em fase experimental, através da produção de um vídeo nos mesmos moldes das vídeo-aulas regulares geradas na instituição, mas com caráter exploratório e demonstrativo. O conteúdo didático dessa vídeo-aula é o procedimento de criação de uma rede adicional para o cluster. Neste estudo de caso, o professor demonstra como criar uma rede local virtual, denominada VLAN, através do switch (dispositivo que gerencia as redes para reencaminhar módulos - frames - entre os diversos nós). Dentro do cluster do CESUP há três redes geridas. Uma rede de alto desempenho, uma de gerência e outra de trabaIho (normal). Para dividir a rede em segmentos menores (como as duas redes de trabalho mostradas na Figura 2), cada rede adicional pode ser criada primeiramente acessando-se a interface de configuração do switch e identificando que portas devem ser utilizadas. 


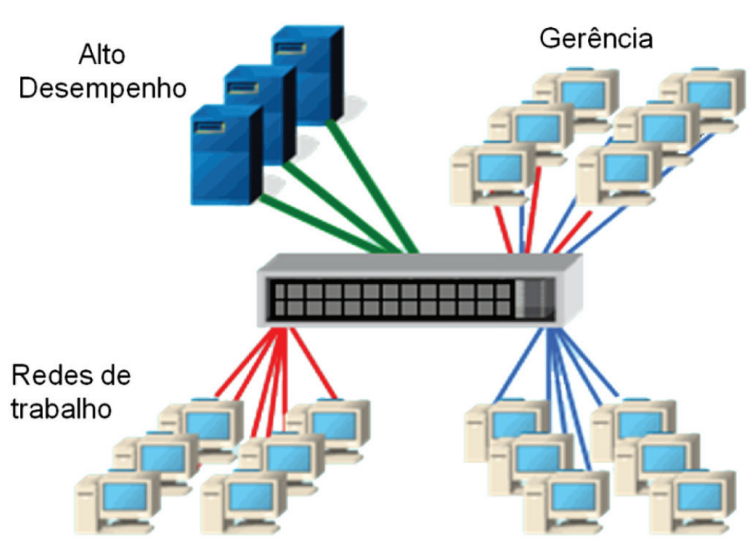

FIGURA 2 - Desenho Esquemático Mostrando o Swith e a Possibilidade de Divisão da Rede em Segmentos Menores.

FONTE: Elaborada pelos autores.

O vídeo pretende apresentar o switch, seu funcionamento e seus componentes, para que o aluno seja capacitado a criar uma rede adicional para a máquina. A produção está sendo feita no estúdio de gravação do CESUP/UFRGS, com captação e edição em tempo real através da ilha de edição (Figura 3). Inicialmente, se produziu um teste de uso da tecnologia, com a utilização e demonstração da RA e dos outros recursos já utilizados regularmente.

Na primeira parte da vídeo-aula demonstrativa, o equipamento em questão foi mostrado através de sua imagem inserida em tela cheia no vídeo; e de sua imagem misturada eletronicamente com a do professor, de forma que este pudesse apontar fisicamente partes e componentes (Figura 4). Essa tecnologia, chamada de chroma-key, é conhecida por sua utilização em programas televisivos de previsão meteorológica, em que o apresentador é mostrado junto ao mapa. Este vídeo foi captado e editado nas instalações do CESUP/UFRGS, utilizando o equipamento já citado, e finalizado no software em software profissional de edição. Está disponível para visualização no canal do
YouTube do CESUP, onde também estão publicadas outras produções realizadas pelo Centro (UNIVERSIDADE FEDERAL DO RIO GRANDE DO SUL, 2010).

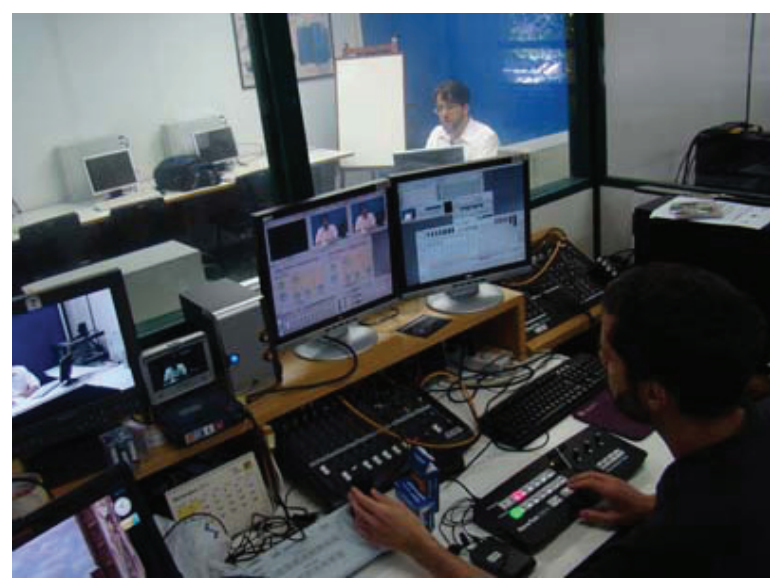

FIGURA 3 - Estúdio de Gravação do CESUP/UFRGS.

FONTE: Elaborada pelos autores.

Num segundo momento, experimentou-se a RA (também abordada no vídeo acima citado), executada através do sistema Sacra versão 2010 (SACRA, 2010). Foram previamente impressos os marcadores, usando os arquivos .pdf disponibilizados e inserido um novo objeto 3D - uma chapa plana modelada com a linguagem VRML através de um arquivo texto com extensão .wrl. Sobre ela, foi aplicada como textura uma foto do switch em formato .jpg. Para elaboração dessa chapa, primeiramente definiu-se um objeto tridimensional (em VRML 2 chamado de Shapes) que em geral contém dois atributos, a Aparência e a Geometria. $\mathrm{Na}$ Aparência definiu-se a textura, no caso a imagem a ser exibida. Já na Geometria definiu-se um objeto em forma de cubo, com as dimensões do painel (largura 10, altura 0.01 e profundidade 7.5). 


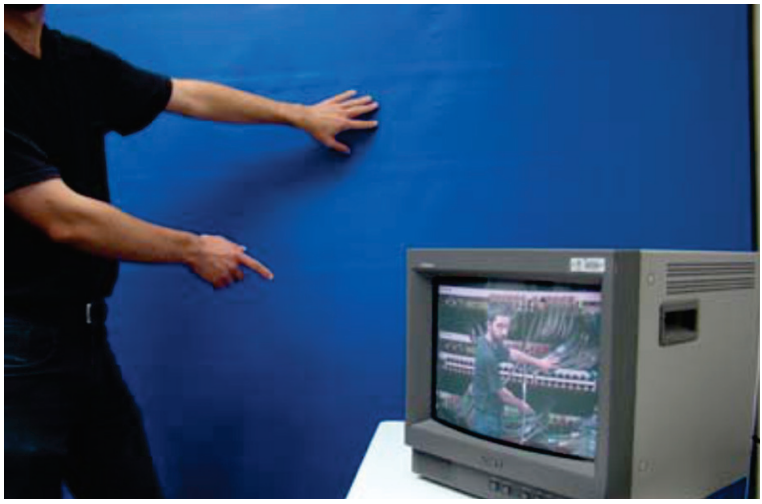

FIGURA 4 - Imagem do Professor Misturada Eletronicamente com a do Equipamento, Através de Chroma-key.

FONTE: Elaborada pelos autores.

Para essa parte do experimento, um computador, dotado de webcam $1.3 \mathrm{M}$, e executando a RA, foi conectado à ilha de captação e edição de vídeo, para receber o sinal que normalmente é gravado e/ou transmitido. As duas câmeras do estúdio de gravação foram utilizadas para capturar a imagem do professor segurando o marcador. Na imagem capta$\mathrm{da} /$ gravada, aparece o professor segurando a fotografia do equipamento em questão, como se ele estivesse manipulando o próprio equipamento e seus componentes (Figura 5).

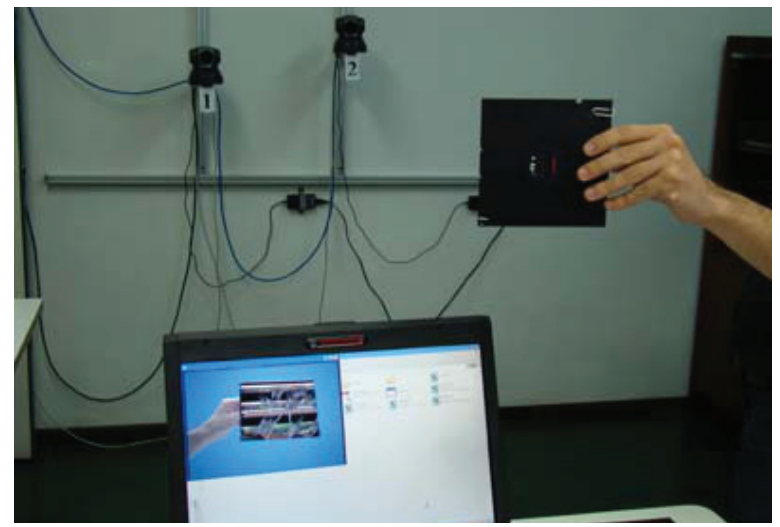

FIGURA 5 - Professor Segurando o Marcador Para a Captura por Câmeras com Controle Remoto e a Imagem do Switch no Monitor. FONTE: Elaborada pelos autores.
Uma variação dessa experiência foi feita através da mesa de apresentação de documentos. Nessa mesa, que normalmente serve para apresentar transparências ou pequenos objetos, foi colocado o marcador e, conforme a Figura 6, a imagem captada/gravada mostra o próprio equipamento em questão, como se estivesse sendo manipulado e apresentado pelas mãos do professor.

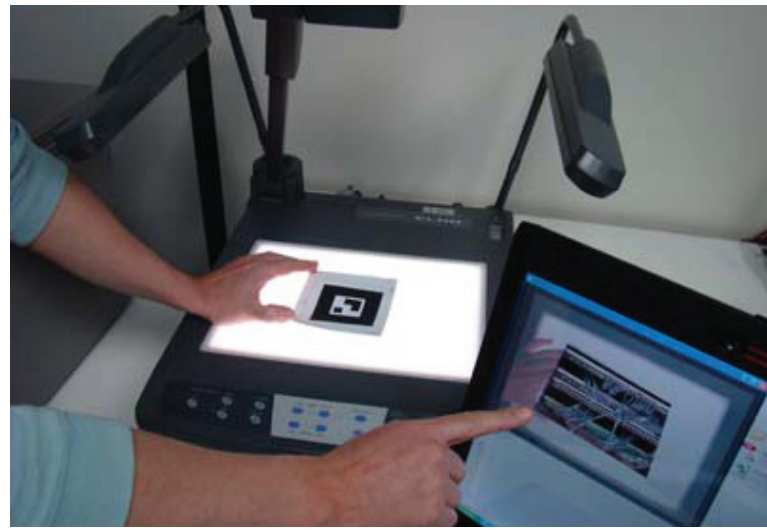

FIGURA 6 - Suporte a Criação de uma Rede Adicional com a Visualização das Portas do Switch Através da RA.

FONTE: Elaborada pelos autores.

Comparando a RA com as outras ferramentas, pôde-se verificar que em relação à mesa de apresentação de documentos, a RA reduz o custo de produção, já que não é necessário imprimir lâminas ou transparências de cada material que se queira colocar na mesa de apresentação para mostrar aos alunos. Comparando com a técnica de chromakey, notou-se que a RA permite uma melhor usabilidade já que com uma pequena variação no desenho dos marcadores é possível incluir pontos que podem funcionar como botões e uma vez que os mesmos sejam tocados, um outro objeto previamente associado o mesmo pode ser mostrado. Ou seja, também seria possível, por exemplo, apresentar uma modificação do mes- 
mo indicando uma sequencia de uma operação de montagem, através da oclusão de um ponto que seria tapado por um dedo. Em todos os casos, a possibilidade de utilização de comando de voz através de um software de reconhecimento, como o Microsoft Speech, poderia permitir uma interação ainda maior.

\section{Apresentação do Cluster Virtual Através da RA no UFRGS Portas Ab- ertas 2012}

Foi realizada uma apresentação do setor de EAD do CESUP e de um modelo virtual do cluster do CESUP através de Realidade Aumentada para o público no evento UFRGS Portas Abertas 2012. O objetivo foi avaliar a relevância do emprego da RA no aprendizado do público (composto em sua maioria de alunos do ensino médio e dos professores responsáveis pelas turmas), através da visualização interativa proveniente do controle manual do posicionamento do conteúdo, neste caso, um modelo tridimensional do cluster do CESUP. Para isso, foi preparado um experimento onde os alunos puderam manipular um marcador previamente associado com este modelo virtual do cluster, de modo que fosse possível interagir, conferindo todas as faces através da projeção no monitor e, assim, facilitar a compreensão dos alunos em relação ao funcionamento do processamento de alto desempenho, bem como do conceito de agrupamento de vários processadores, conectados através de diversos meios de comunicação físicos e lógicos, operando em conjunto, como se fossem uma única máquina.

Para fazer a modelagem do cluster do CESUP, foram tiradas diversas fotos do cluster real e feita a montagem e edição das mesmas através do software Photoshop para produzir a imagem a ser utilizada como textura do cluster virtual. $\mathrm{O}$ arquivo .wrl gerado pode ser utilizado através de programas para visualização de arquivos como o Cortona ou o Vivaty, ou através da alguma biblioteca de realidade aumentada (RA) como o ARToolKit, que foi a utilizada neste trabalho. Foi desenvolvido um marcador personalizado com o nome do CESUP sendo o mesmo associado ao cluster modelado (arquivo de extensão .wrl) através da execução de um utilitário de reconhecimento que faz parte do pacote ARToolKit. Além disso, foi compilado um executável contendo todos os arquivos necessários para visualizar o cluster em RA. A Tabela 1 lista as etapas com figuras ilustrativas dos cinco passos necessários para este propósito.

\begin{tabular}{|l|}
\hline $\begin{array}{l}\text { 1. Abrir o arquivo marca- } \\
\text { dor (marcador.pdf); }\end{array}$ \\
\hline $\begin{array}{l}\text { 2. Imprimir o marcador } \\
\text { (marcador.pdf) e colar em } \\
\text { um material rígido como } \\
\text { cartolina ou papelão; }\end{array}$ \\
\hline $\begin{array}{l}\text { 3. Certificar-se que a } \\
\text { webcam esta instalada } \\
\text { corretamente e acionar o } \\
\text { executável (cluster.exe); }\end{array}$ \\
\hline $\begin{array}{l}\text { 4. Depois de aparecer } \\
\text { a tela de vídeo, colocar } \\
\text { o marcador em frente à } \\
\text { webcam. Sobre o marca- } \\
\text { dor no monitor, aparecerá } \\
\text { o cluster. Caso contrário, } \\
\text { variar as condições de } \\
\text { iluminação e movimentar } \\
\text { o marcador lentamente. }\end{array}$ \\
\hline
\end{tabular}

TABELA 1: Procedimento Para Visualização do Cluster em RA.

FONTE: Elaborada pelos autores. 
A Figura 7A mostra uma visualização do arquivo .wrl produzido sendo executado através do aplicativo de RA. Na Figura 7B é mostrada uma falha comum de ocorrer e que foi explicada e demonstrada para os alunos. Ela é denominada oclusão e atrapalha a visualização da imagem quando partes do marcador são escondidas. Isso faz com que o padrão do marcador não seja mais reconhecido e o cluster virtual relacionado a esse marcador desaparece. Outra limitação possível é se o marcador não estiver completamente visível para a câmera.

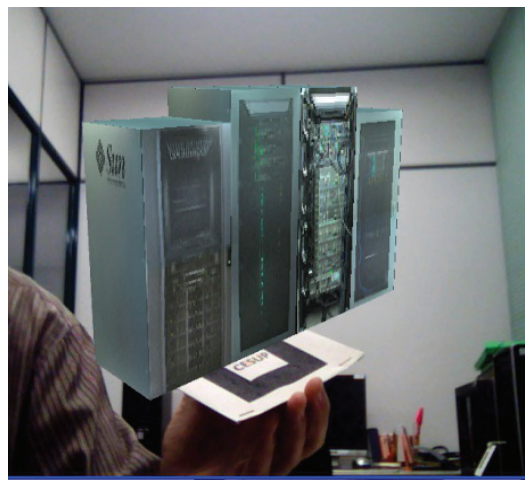

FIGURA 7A - Cluster Virtual Sendo Visualizado no Aplicativo de RA. FONTE: Elaborada pelos autores.

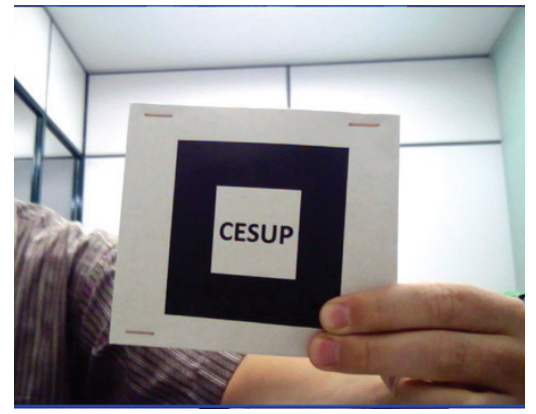

FIGURAS 7B - Cluster Virtual Sendo Visualizado no Aplicativo de RA sem Aparecer Devido à Obstrução com os Dedos.

FONTE: Elaborada pelos autores.

Em comparação com os softwares apenas de visualização dos arquivos .wrl, o emprego da RA mostrou ser um recurso computacional que possibilita maior interação com o conteúdo. Enquanto nos aplicativos de visualização esta se da apenas com comandos no teclado, onde o controle preciso dos movimentos requer um nível elevado de coordenação motora, a visualização do cluster através da RA permite a manipulação direta, podendo ser intuitivamente girado e aproximado manualmente pelos usuários.

Com relação ao feed back dos alunos sobre a visualização do Cluster através da RA, aproximadamente $90 \%$ dos mesmos responderam que fica mais fácil compreender os conceitos teóricos abordados por meio da interação proporcionada por este recurso. Além disso, alunos comentaram que se sentiram atraídos a participarem de forma mais ativa, o que demonstrou que o emprego da RA neste estudo de caso de construção do conhecimento foi significantemente positiva.

\section{Conclusão}

Neste trabalho foi feito um estudo sobre o uso da realidade aumentada como ferramenta auxiliar às demais tecnologias educacionais com o objetivo de explorar o potencial de contribuição desta tecnologia na construção do conhecimento no ensino presencial e à distância. Foram feitos experimentos dentro da estrutura já existente no CESUP/UFRGS com câmeras controladas com joystick e através de uma mesa de apresentação de documentos. Foi produzido um vídeo de caráter exploratório e demonstrativo sobre o uso da RA como tecnologia educacional para a criação de uma rede adicional para o cluster do CESUP/UFRGS.

O uso da RA integrada ao setor de EAD do CESUP comparou a RA com as outras ferra- 
mentas disponíveis. Quando comparada à técnica que emprega a mesa de apresentação de documentos, a RA dispensa as despesas com impressão de transparências para cada material que se bote na mesa para mostrar aos alunos. Confrontando com a técnica de chromakey, observou-se que a RA permite uma melhor usabilidade e interatividade já que com pequenas variações nos desenhos dos marcadores é possível incluir pontos para funcionarem como botões, de modo que, uma vez que os mesmos sejam tocados, outro objeto previamente associado pode ser visualizado também.

O artigo abordou ainda a experiência de apresentação do modelo virtual do cluster do CESUP através da Realidade Aumentada para o público no evento UFRGS Portas Abertas 2012. Nesta ocasião explicou-se como o marcador personalizado com o nome do CESUP foi associado ao cluster modelado e o procedimento necessário para visualizar o cluster em RA. Foram feitas demonstrações para os alunos onde mesmos puderam interagir na visualização do arquivo produzido através do aplicativo de RA. Também foram explicadas e mostradas duas limitações relacionadas a esta demonstração e que provocaram o desaparecimento do cluster virtual do monitor: a necessidade do marcador estar completamente visível para a câmera e a falha de oclusão que pode ocorrer se alguma parte do marcador ficar escondida. Os experimentos mostraram que o emprego da RA na área de educação e treinamento apresenta um grande potencial de auxiliar o processo de construção do conhecimento dos usuários através da visualização e a manipulação de objetos virtuais modelados por computador no mundo real.

\section{Agradecimentos}

Ao CNPQ, aos colegas do CESUP, em especial ao técnico Lindolfo Meira e a diretora Denise Grüne Ewald, e ao professor Renato Ventura Bayan Henriques e demais colegas do Departamento de Engenharia Elétrica da UFRGS.

\section{Referências}

ARTAG. [S.I.: s.n.], 2009. Disponível em: <http://www.artag.net/> Acesso em: 27 ago. 2010.

ARTOOLKIT. [SI.: s.n.], [2005?]. Disponível em: <http://www.hitl.washington.edu/artoolkit/> Acesso em: 18 out. 2005.

AZUMA, R.T. A Survey of Augmented Reality. Presence: teleoperators and virtual environments, Cambridge, Mass., v. 6, n. 4, p. 355-385, 1997.

AZUMA, R.T. et al. Recent Advances in Augmented Reality. IEEE Computer Graphics \& Applications, Washington, DC, v. 21, n. 6, p. 34-47, nov./dez. 2001. 
BAJURA, M.; NEUMANN, U. Dynamic Registration Correction in Video-Based Augmented Reality Systems. IEEE Computer Graphics \& Applications, Washington, DC, v. 15, n. 5, p. 52-60, 1995.

BERNARDINO A. JUNIOR, A.; CARDOSO, A.; LAMOUNIER Jr., E. Estudo de Máquinas Elétricas Auxiliado por Técnicas de Realidade Virtual. Uberlândia: Universidade Federal de Uberlândia/Faculdade de Engenharia Elétrica, [2000?]. Disponível em: <http://www2.fc.unesp.br/wrva/artigos/50081.pdf> Acesso em: 27 ago. 2010.

BLUEMEL, E. et al. Virtual Environments for the Training of Maintenance and Service Tasks. In: SIMULATION CONFERENCE, 2003, New Orleans, LA. Proceedings 2003. Washington, DC: IEEE, 2003. v. 2, p. 2001-2007.

BOUD, A.C. et al. Virtual Reality and Augmented Reality as a Training Tool for Assembly Tasks. In: INTERNATIONAL CONFERENCE ON INFORMATION AND VISUALIZATION, 1999, London. Proceedings. Washington, DC: IEEE, 1999. V. 4, p. 32-36.

COMPORT, A.I.; MARCHAND, E.; CHAUMETTE, F. A real-time tracker for markerless augmented reality. In: INTERNATIONAL SYMPOSIUM ON MIXED AND AUGMENTED REALITY, 2003, Tokyo. Proceedings. Washington, DC: IEEE, 2003. P. 36- 45.

CRISPEN. [S.I.: s.n.], [2010?]. Disponível em: <http://vrmlworks.crispen.org/history.html/> Acesso em: 18 ago. 2010.

CUNHA, K.K.C.; GUIMARÃES, M.P. Desenvolvimento de uma Interface Gráfica Para o Artoolkit com Aplicação na Área Educacional. São Paulo, 2007. Trabalho apresentado no WRVA'07 Workshop de Realidade Virtual e Aumentada, em 2007, Itumbiara, BR-GO. Disponível em: <http://www.ckirner. com/sacra/> Acesso em: 27 ago. 2010.

ESPÍNDOLA, D.B. et al. Using Mixed Reality in the Visualization of Maintenance Processes. In: IFAC Workshop on Advanced Maintenance Engineering, Services and Technology, 1., Lisboa, 2010. Advanced Maintenance Engineering. Lisboa: Universidade Nova de Lisboa, 2010. V. 1, pt. 1, p. 30-35. FEINER, S.; MACINIYRE, B.; SELIGMANN, D. Knowledge based Augmented Reality. Communications of the ACM, New York, v. 36, n, 7, p. 53-62, jul. 1993.

FIALA, M. ARTag, a Fiducial Marker System Using Digital Techniques. Computer Vision and Pattern Recognition, Washington, DC, v. 2, p. 590-596, 2005.

FORTE, C. et al. Implementação de Laboratórios Virtuais em Realidade Aumentada para Educação à Distância. In: WORKSHOP DE REALIDADE VIRTUAL E AUMENTADA, 5., 2008, Bauru, BR-SP. Anais. Bauru: Ed. UNESP, 2008. V. 1, p. 20-28. 
HULF, R. et al. Usando Iluminação Baseada em Imagens na Geração de Ambientes de Realidade mista. Porto Alegre, 2004. Trabalho apresentado no Workshop sobre Realidade Aumentada, em maio de 2004, Piracicaba, BR-SP. Disponível em: <http://www.lbd.dcc.ufmg.br/colecoes/svr/2004/ aumentada3.pdf> Acesso em: 27 jan. 2011.

KIRNER, C. Mãos Colaborativas em Ambientes de Realidade Misturada. In: WORKSHOP SOBRE REALIDADE AUMENTADA, 1., 2004, Piracicaba. Anais do WRA'2004. Piracicaba: UNIMEP, 2005. P. 1-4.

KIRNER, C.; SANTIN, R. Funcionamento e Utilização do Sistema de Autoria Colaborativa com Realidade Aumentada - SACRA. [S.I.: s.n.], 2010.

KLOPFER, E. Augmented Learning: research and design of móbile educational games. Cambridge, Mass.: The MIT Press, 2008.

LI, J.R.; KHOO, L.P.; TOR, S.B. Desktop Virtual Reality for Maintenance Training: an Object Oriented Prototype System (V-REALISM). Computers in Industry, Amsterdam, v. 52, p. 109-125, 2003.

MILGRAM, P. et al. Augmented Reality: a class of displays on the reality- virtuality continuum. SPIE Telemanipulator and Telepresence Technologies, Boston, MA, v. 2351, p. 282-292, 1994.

PROVIDELO, C. et al. Ambiente Dedicado Para Aplicações Educacionais Interativas com Realidade Misturada. São Carlos, 2004. Trabalho apresentado no Workshop sobre Realidade Aumentada, em maio de 2004, Piracicaba, BR-SP. Disponível em: <http://www.academia.edu/617349/Ambiente_dedicado_para_aplica\%C3\%A7\%C3\%B5es_educacionais_interativas_com_Realidade_Misturada> Acesso em: 27 jan. 2011.

SACRA: Sistema de Autoria Colaborativa com Realidade Aumentada. [S.I.: s.n.], 2010. Disponível em: <http://www.ckirner.com/sacra/SACRA-2010.zip> Acesso em: 27 ago. 2010.

SISCOUTO, R.A.; TORI, R. AVTC - Augmented Virtuality Tele-Conferencing. São Paulo, 2004. Trabalho apresentado no Workshop sobre Realidade Aumentada, em maio de 2004, Piracicaba, BR-SP. Disponível em: <http://www.lbd.dcc.ufmg.br/colecoes/svr/2004/aumentada2.pdf> Acesso em: 27 jan. 2011.

TIMM, M.I. et al. Gestão de EAD: projeto de infraestrutura e atividades de planejamento. [S.I.:s.n.], 2008. Trabalho apresentado no Congresso Brasileiro de Ensino Superior à Distância, 5., 2008, Gramado, BR-RS.

TRIGO, C.H.; KIRNER, C. Incorporando Colaboração com Realidade Virtual e Aumentada em Ambientes de Educação à Distância. São Paulo, 2007. Trabalho apresentado no WRVA'07 Workshop 
de Realidade Virtual e Aumentada, em 2007, Itumbiara, BR-GO.

UNIVERSIDADE FEDERAL DO RIO GRANDE DO SUL. Centro Nacional de Supercomputação. CESUP Centro Nacional de Supercomputação [homepage]. Porto Alegre, 2010. Disponível em: <http:// www.youtube.com/user/cesupUFRGS> Acesso em: 27 jan. 2011.

UNIVERSIDADE FEDERAL DO RIO GRANDE DO SUL. Centro Nacional de Supercomputação. CESUP Centro Nacional de Supercomputação [homepage]. Porto Alegre, 2011. Disponível em: <http:// www.cesup.ufrgs.br/> Acesso em: 27 jan. 2011.

Submetido para avaliação em 04 de março de 2011.

Aprovado para publicação em 14 de junho de 2014.

Eduardo Luis Schneider: Universidade Federal do Rio Grande do Sul - Porto Alegre - RS - Brasil. E-mail: edu. ufrgs@gmail.com

André Soares Grassi: Universidade Federal do Rio Grande do Sul - Porto Alegre - RS - Brasil. E-mail: asgrassi@ gmail.com

Leonardo Bisch Piccoli: Universidade Federal do Rio Grande do Sul - Porto Alegre - RS - Brasil. E-mail: Ibpiccoli@ gmail.com

Maria Isabel Timm: Universidade Federal do Rio Grande do Sul - Porto Alegre - RS - Brasil. E-mail: beta@cesup. ufrgs.br 